\title{
East South Central States Census Division
}

National Cancer Institute

\section{Source}

National Cancer Institute. East South Central States Census Division. NCI Thesaurus. Code C76343.

A census division of the United States consisting of Alabama, Kentucky, Mississippi, and Tennessee. 